\title{
Avaliação do uso do modelador nasal em pacientes portadores de fissura labial unilateral operados pela técnica de Göteborg
}

\author{
Evaluation of the nasal retainer in patients with unilateral cleft lip operated upon \\ by Göteborg technique
}

Celso Luiz Buzzo ${ }^{1}$

Diogo Franco ${ }^{2}$

Eudes Soares de Sá

Nobrega $^{3}$

Renato da Silva-Freitas ${ }^{4}$

Cesar Augusto Adami

RAPOSO DO AMARAL ${ }^{5}$

Daniel Rogge Carone 5

Trabalho realizado no Hospital da SOBRAPAR - Sociedade Brasileira de Pesquisa e Assistência para a Reabilitação Craniofacial, Campinas, SP, Brasil.

Trabalho vencedor do Prêmio Antonio Prudente 2010.

Artigo submetido pelo SGP (Sistema de Gestão de Publicações) da RBCP.

Artigo recebido: 24/7/2010 Artigo aceito: 25/11/2010

\begin{abstract}
RESUMO
Introdução: Após avaliação da técnica empregada em nosso serviço para correção de fenda labial unilateral, observamos uma pontuação negativa maior com relação ao resultado final do nariz, o que nos levou a manter nossa linha de pesquisa, com o objetivo de introduzir e avaliar novo método para moldagem nasal pós-operatória imediata, por meio de molde de silicone. Método: Foram avaliados 20 pacientes operados pela mesma técnica, sendo que 10 deles utilizaram o molde nasal, e os outros 10 não. A avaliação foi feita por três cirurgiões de grande experiência, utilizando fotos pré e pós-operatórias e um filme do paciente com sua idade atual, sem o conhecimento de qual grupo utilizou o molde. Resultados: A idade de avaliação no período pós-operatório variou de 1 a 9 anos, do procedimento cirúrgico; somente $60 \%$ dos pacientes foram operados na idade preconizada pelo nosso protocolo (3 meses), o tempo médio de uso do modelador foi de 4 meses. Após a aplicação de análise estatística, observamos que não houve melhora de resultados nos pacientes que usaram o modelador nasal, em comparação ao grupo que não usou. Conclusões: $\mathrm{O}$ grupo de pacientes operados pela técnica de Göteborg com a utilização do modelador nasal não apresentou resultados melhores estatisticamente significantes quando comparado ao grupo de pacientes operados pela mesma técnica sem o uso do modelador nasal.
\end{abstract}

Descritores: Fenda labial. Lábio/cirurgia. Nariz/anormalidades/cirurgia.

\begin{abstract}
Introduction: After evaluating the technique chosen for unilateral cleft lip surgery, we notice a negative outcome of the nose aspect, what lead us to introduce and evaluate a new method of nasal molding with a silicone strut. Methods: There were evaluate 20 patients operated by the same technique, 10 patients with immediate silicone strut molding and 10 patients without nasal molding. The results were analyzed with pre-operatory pictures, post operative pictures and a movie of the child in the present date by three expertise cleft lip surgeon, whose did not know which use or not the silicone molding. Results: The age of the patients after the surgery range from 1 to 9 years, which only $60 \%$ were operated on the pre set age ( 3 months), the average time using the Silicone molding strut were 4 months. After statistics analysis, it demonstrated that there was not a better outcome in the nose aspect on patients who's used the nasal molding. Conclusions: After statistics analysis, the group of patients underwent the Göteborg Technique, using immediate silicone strut molding, shown no better results than the group without nasal molding.
\end{abstract}

Keywords: Cleft lip. Lip/surgery. Nose/abnormalities/surgery.

1. Mestre em cirurgia; titular da SBCP, Regente do Serviço Integrado de Cirurgia Plástica "Prof. Dr. Cássio M. Raposo do Amaral”, Campinas, SP, Brasil.

2. Professor Adjunto da Universidade Federal do Rio de Janeiro, Rio de Janeiro, RJ, Brasil.

3. Cirurgião Plástico do "Centrinho" de Bauru, Bauru, SP, Brasil.

4. Professor Adjunto da Universidade Federal do Paraná, Curitiba, PR, Brasil.

5. Médico residente da SOBRAPAR - Sociedade Brasileira de Pesquisa e Assistência para a Reabilitação Craniofacial, Campinas, SP, Brasil. 


\section{INTRODUÇÃO}

Patologia de maior frequência dentre as deformidades congênitas craniofaciais, a fissura labiopalatina causa grandes distorções anatômicas no lábio superior, nariz e palato.

As alterações na fala e as possíveis sequelas estéticas que poderão surgir, caso o tratamento não seja aplicado de forma eficaz e no tempo adequado, tornam esta afecção merecedora de atenção especial do cirurgião e de toda equipe multidisciplinar envolvida em seu tratamento. Em 1995, recebemos na SOBRAPAR - Sociedade Brasileira de Pesquisa e Assistência para a Reabilitação Craniofacial - a visita dos professores Dr. Bengt Johanson, Dr. Jan Ljla, cirurgiões plásticos, e do Dr. Hans Fried, ortodontista, oriundos da cidade de Göteborg, Suécia. Iniciava-se aí um convênio científico-cultural entre a SOBRAPAR e a Universidade de Göteborg, coordenado pelo Prof. Dr. Cassio M. Raposo do Amaral e pelo Prof. Dr. Bengt Johanson, com o objetivo de pesquisar e desenvolver técnicas cirúrgicas para o tratamento das fissuras labiopalatinas.

Foi nesta ocasião que tivemos contato com a técnica de labioplastia desenvolvida na cidade de Göteborg, a qual praticamos durante todos estes anos, até que, no ano de 2000, padronizamos como técnica operatória em nosso protocolo para tratamento da fissura labial unilateral e passamos a denominá-la como técnica de Göteborg ${ }^{1}$.

\section{Histórico}

Desde os tempos mais remotos, observamos a preocupação dos povos com relação a esta patologia tão deformante. Esculturas e manuscritos encontrados por arqueólogos comprovam tal afirmação. O comportamento da sociedade frente a esta patologia varia abruptamente, dependendo da origem étnica, costumes, religião, etc.

Fatos tristes ocorreram na Idade Média, durante a Inquisição, quando crianças fissuradas eram mortas pelas próprias mães que temiam serem queimadas vivas. Fatos semelhantes a estes ainda ocorrem em algumas tribos indígenas em nosso país ${ }^{1}$.

Desde 390 a.C. na China, quando se relatou o sucesso do fechamento de uma fissura labial, inúmeras técnicas surgiram para o tratamento da fissura labial. Ambroise Paré, em 1564, descreveu uma técnica cirúrgica onde transfixava as vertentes labiais previamente incisadas, com uma longa agulha, e as aproximava com fios ancorados nesta agulha ${ }^{2,3}$.

Rose, em 1891, e Thompson, em 1912, descreveram técnica semelhante, utilizando incisões anguladas em toda a borda das vertentes labiais, de modo que, durante a sutura, haveria um alongamento deste lábio, tentando-se evitar os "entalhes" no vermelhão. Surgem, então, técnicas utilizandose retalhos com o objetivo de alongar o lábio e evitar retrações cicatriciais tão comuns nas cicatrizes lineares, tentando-se manter a simetria labial e o "arco de cupido" mais natural ${ }^{2-4}$.

Mirault, em 1844, e Giraldes, em 1866, publicaram técnicas utilizando-se retalhos em forma triangular; em 1949, Le Mesurier, baseado em procedimento descrito por Hagedom em 1892, apresentou uma técnica utilizando a transposição de retalhos quadrangulares.

Nomes como Tennison, Randall e Skoog, entre outros, devem ser destacados como ícones na evolução do tratamento da fissura labial, assim como gostaríamos de enfatizar os nomes de cirurgiões brasileiros como Perseu Lemos e Vitor Spina, tão bem lembrados em recente publicação na Revista da Sociedade Brasileira de Cirurgia Plástica pelo Dr. Jaime Anger ${ }^{2,4,5}$.

Coube, entretanto, a Ralph Millard Jr. desenvolver uma técnica utilizando um retalho de rotação e avanço em 1955, sendo publicada pela primeira vez em 1957, difundindose rapidamente por todo o mundo, pela simplicidade de sua confecção e pelos bons resultados apresentados. Após algumas modificações feitas pelo próprio Millard, dentre as quais a atuação nas cartilagens nasais no mesmo tempo cirúrgico da labioplastia primária, corrigindo-se assimetrias narinárias com excelentes resultados, esta técnica difundiu-se rapidamente e hoje é realizada em vários centros do mundo ${ }^{1}$.

Harold McComb, em 1985 e 1986, e Keneth Salyer, em 1986, observaram que não houve alteração no crescimento e no desenvolvimento das cartilagens nasais quando efetuada a rinoplastia durante a cirurgia primária do lábio (labiorrinoplastia), vindo a justificar o entusiasmo de vários cirurgiões com relação a este procedimento ${ }^{4,6,7}$.

Procedimento este também adotado em nossa técnica cirúrgica, com ampla abordagem da cartilagem alar do lado fissurado, liberando-a completamente e também com amplo descolamento do todo o dorso nasal, com posterior "modelagem" das estruturas nasais, com pontos captonados.

Tendo em vista a avaliação dos resultados de nosso trabalho apresentado no Congresso Brasileiro de Cirurgia Plástica, em 2007, na cidade de Curitiba, PR, Brasil, onde avaliamos o resultado final baseados em 3 itens: cicatriz labial, linha cutâneo-mucosa e nariz, observamos neste último uma pontuação negativa maior ${ }^{1}$.

Estimulados pela procura de um resultado melhor e mantendo nossa linha de pesquisa, iniciamos novo método cirúrgico em que passamos a utilizar um modelador nasal de silicone pré-moldado (SILIMED), colocado no final da cirurgia e mantido por um período de 6 meses.

Dentro desse contexto, o objetivo principal desse trabalho é avaliar a influência do uso de modeladores nasais de silicone no formato nasal e no conjunto labial na técnica de Göteborg, no período pós-operatório, sendo o tempo máximo de uso do modelador de 6 meses.

\section{MÉTODO}

O projeto foi aprovado pela Comissão de Ética Médica do hospital da Sobrapar. 
Foram avaliados 20 pacientes operados no período de janeiro de 2001 a março de 2009, todos operados pelo autor ou pelo residente mais graduado com supervisão direta do mesmo, seguindo-se protocolo da Sobrapar para pacientes portadores de fissuras labiopalatinas. Dez pacientes utilizaram o modelador nasal e os outros 10 não o utilizaram.

O protocolo indica idade ideal para cirurgia aos 3 meses, peso mínimo de 4,5 kg, hemoglobina com um mínimo de 10 $\mathrm{mg} \%$, associado aos exames de rotina e avaliação clínica préanestésica. Devido às condições socioeconômicas do nosso país, o que influencia diretamente na avaliação do quadro nutricional, os pacientes que estavam acima da idade ideal, com no máximo 6 meses, também foram incluídos neste estudo. Os critérios de exclusão foram: idade superior a 6 meses e ausência de documentação fotográfica adequada.

A avaliação dos resultados foi realizada por três cirurgiões com ampla experiência com este tipo de patologia.

Foram enviadas aos avaliadores, fotografias pré e pósoperatórias, acrescentado por um vídeo com tempo médio de 2 minutos, no qual os pacientes, quando a idade o permitia, pronunciavam algumas palavras e adotavam algumas posições para melhor avaliação pós-operatória. Também foi enviada tabela padronizada para preenchimento dos resultados apontados. Os avaliadores não foram informados quais pacientes haviam utilizado o modelador nasal e solicitamos aos mesmos que utilizassem o método proposto pela American Cleft Palate Association ${ }^{10}$, que avalia a gravidade pré-operatória e o resultado pós-operatório, padronizando e facilitando a avaliação, dando ênfase ao nariz de forma isolada.

O Quadro 1 sintetiza o método de avaliação proposto pela American Cleft Palate Association.

A análise estatística foi realizada utilizando-se o software SPSS V 16, Minitab 15 e Excel Office 2007.

\section{Análise Estatística}

Devido à pequena amostragem, definimos como nível de significância $0,10(10 \%)$, um pouco acima do usualmente utilizado (5\%), sendo o nosso objetivo comparar os grupos operados com e sem o tutor nasal.

\section{RESULTADOS}

Foram avaliados 20 pacientes, sendo $14(70 \%)$ do sexo masculino, e $6(30 \%)$ do feminino. Com relação ao tipo da fissura, a fissura labiopalatina direita foi a mais frequente $(45 \%)$, as fissuras labial esquerda e labiopalatina esquerda apresentaram-se de forma equilibrada $(20 \%)$ e, por último, a fissura labial à direita (15\%). Somente $12(60 \%)$ pacientes foram operados na idade ideal preconizada em nosso protocolo, 3 meses, o restante variou até a idade de, no máximo, 6 meses.

O tempo médio de uso do modelador nasal foi de 4 meses, sendo necessária em 5 pacientes a recolocação dos modeladores, quando saíram em um tempo inferior a 3 meses, metade do tempo preconizado. A idade para avaliação pós-operatória variou entre 1 ano e 9 anos do procedimento cirúrgico.

As Figuras 1 a 4 apresentam aspectos do pós-operatório imediato.

A avaliação individual de cada cirurgião está relacionada nas Tabelas 1 a 3 .

Devido aos diversos níveis de resposta, decidimos reagrupar os resultados em apenas Bom (excelente, muito bom e bom) e Ruim (satisfatório e pobre). Utilizando-se o teste de igualdade de Duas Proporções, verificamos que embora existam diferenças as mesmas não podem ser consideradas estatisticamente significantes (Tabela 4 e Figura 5).

\section{DISCUSSÃO}

A técnica cirúrgica utilizada nos favorece quando comparada à ampla abordagem das cartilagens do nariz, preceito este defendido por $\mathrm{McComb}^{7}$ e já utilizado também em outras técnicas de labioplastia. Sendo assim, a modelagem nasal se faz de forma eficaz com resultados duradouros. A utilização de um modelador nasal de silicone nos dá maior segurança quanto às retrações cicatriciais que poderão ocorrer no intróito narinário e também no conduto interno, mais precisamente junto às cartilagens triangulares, responsáveis pelo mecanismo "valvular" do nariz. Infelizmente, este trabalho limitou-se a avaliar os resultados estéticos e não funcionais, devido à idade dos pacientes, o que impediu a utilização de métodos para rinometria.

A avaliação dos resultados, feita por três cirurgiões experientes por meio de fotografias e de um breve filme na idade atual dos pacientes, foi inédita e nos pareceu favorável, contribuindo para o aperfeiçoamento da técnica, embora não tenham sido realizadas observações diretas de cada caso.

$\mathrm{O}$ resultado estatístico particularmente nos causou certa

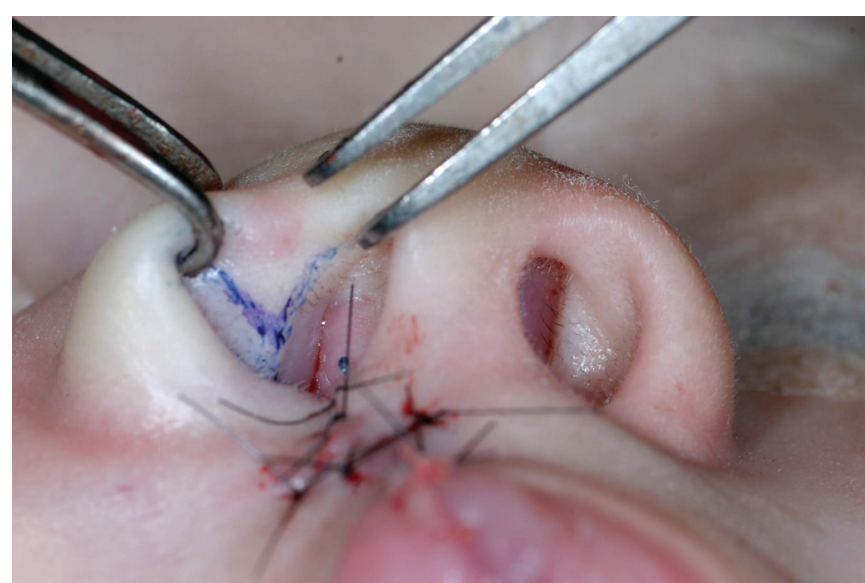

Figura 1 - Pós-operatório imediato sem modelador nasal. 


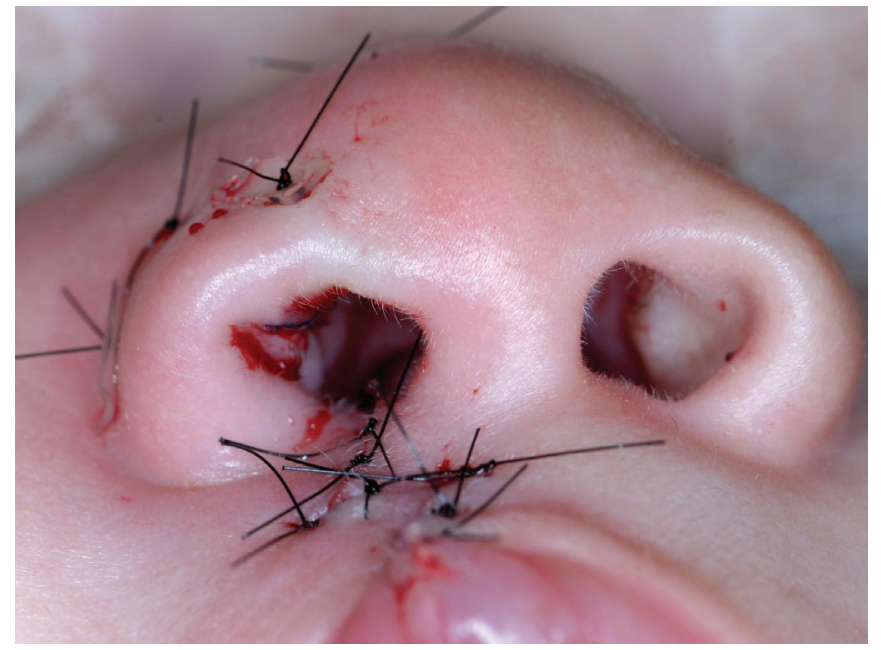

Figura 2 - Pós-operatório imediato sem modelador nasal.

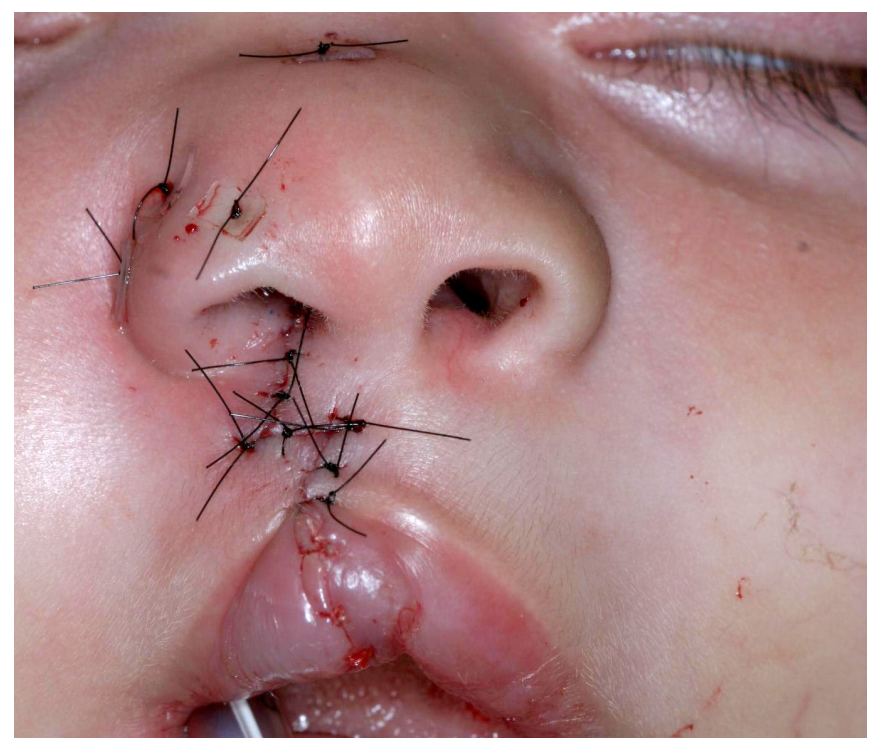

Figura 3 - Pós-operatório imediato sem modelador nasal.

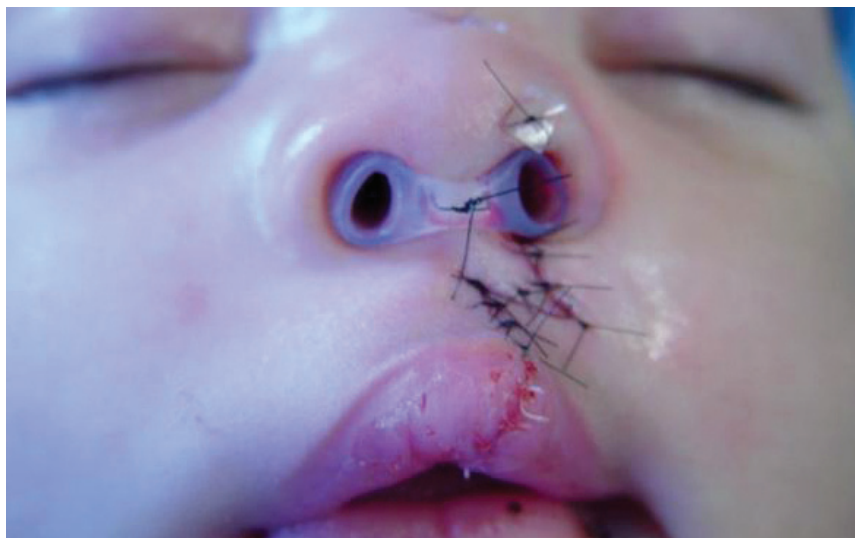

Figura 4 - Pós-operatório imediato com modelador nasal.

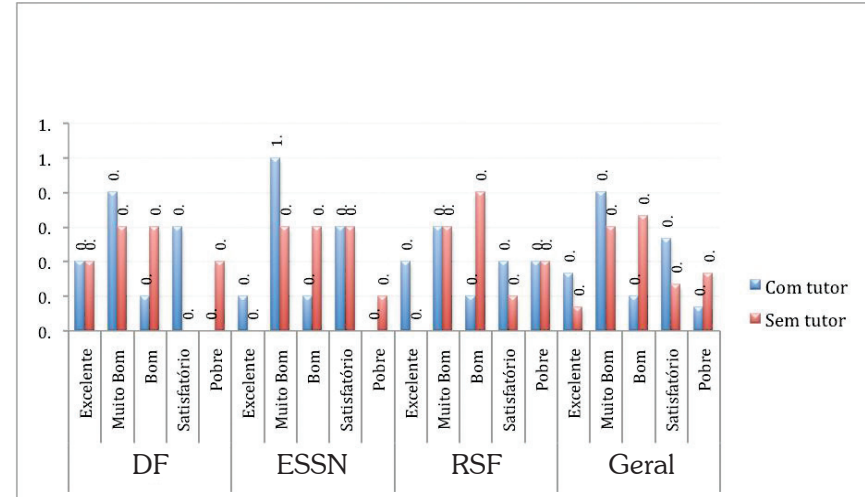

Figura 5 - Comparação com e sem tutor nasal: pós-operatório.

Quadro 1 - Método de avaliação proposto pela American Cleft Palate Association.

\begin{tabular}{l|c}
\hline Categoria & Pontuação \\
\hline Avaliação pré operatória & \\
\hline Leve & $1-3$ \\
Moderado & $4-6$ \\
Severo & $7-11$ \\
Muito severo & $12-16$ \\
\hline Avaliação pós-operatória & \\
\hline Excelente & $0-1,5$ \\
Muito bom & $2-3,5$ \\
Bom & $4-5,5$ \\
Satisfatório & $6-8$ \\
Pobre & $8,5-16$ \\
\hline
\end{tabular}

frustração, pois no acompanhamento pós-operatório observamos resultado melhor nos pacientes que utilizaram o modelador nasal, como podemos observar na Figura 5. Os resultados excelentes foram mais prevalentes entre os pacientes que utilizaram o modelador nasal, já os resultados pobres foram mais frequentes entre aqueles que não utilizaram o modelador nasal.

Também não conseguimos avaliar a parte funcional, o que acreditamos ser muito importante, pois as retrações cicatriciais que ocorrem no conduto narinário são frequentes, atingindo as cartilagens triangulares, responsáveis pela manutenção do mecanismo valvular do nariz. Como observamos, o modelador nasal possui um prolongamento que mantém pérvio este conduto em todo o trajeto, onde estão as cicatrizes, opondo-se às retrações que poderão levar ao estreitamento deste conduto.

É importante destacar que o modelador é pré-moldado, não levando em consideração a variação étnica e o formato das narinas, características de cada raça. Sua aplicação é fácil, a manutenção e a limpeza quando feita por uma mãe 
Tabela 1 - Avaliação do examinador 1 (DF).

\begin{tabular}{|c|c|c|c|c|c|c|c|c|c|}
\hline \multirow[t]{2}{*}{ Paciente } & \multicolumn{4}{|c|}{ Pré-operatório } & \multicolumn{5}{|c|}{ Pós-operatório } \\
\hline & Leve & Moderado & Severo & $\begin{array}{l}\text { Muito } \\
\text { severo }\end{array}$ & Excelente & $\begin{array}{c}\text { Muito } \\
\text { bom }\end{array}$ & Bom & Satisfatório & Pobre \\
\hline P- 5171 & XXX & & & & XXX & & & & \\
\hline B -5532 & & $\mathrm{XXX}$ & & & & XXX & & & \\
\hline H-5694 & & XXX & & & & & XXX & & \\
\hline $\mathrm{V}-5822$ & & & $\mathrm{XXX}$ & & & & $\mathrm{XXX}$ & & \\
\hline B-5876 & & $\mathrm{XXX}$ & & & & & & & $\mathrm{XXX}$ \\
\hline P-6110 & $\mathrm{XXX}$ & & & & XXX & & & & \\
\hline I-6538 & & & $\mathrm{XXX}$ & & & & & & $\mathrm{XXX}$ \\
\hline G-6753 & & & & $\mathrm{XXX}$ & & XXX & & & \\
\hline M-7798 & & & & $\mathrm{XXX}$ & & & $\mathrm{XXX}$ & & \\
\hline P-8127 & XXX & & & & & XXX & & & \\
\hline W-8410 & $\mathrm{XXX}$ & & & & $\mathrm{XXX}$ & & & & \\
\hline H-8477 & & $\mathrm{XXX}$ & & & & & $\mathrm{XXX}$ & & \\
\hline V-8586 & & XXX & & & & & & $\mathrm{XXX}$ & \\
\hline $\mathrm{J}-8840$ & & & $\mathrm{XXX}$ & & & & & $\mathrm{XXX}$ & \\
\hline Y-9107 & & $\mathrm{XXX}$ & & & & $\mathrm{XXX}$ & & & \\
\hline M-9281 & & & $\mathrm{XXX}$ & & & $\mathrm{XXX}$ & & & \\
\hline M-9415 & & & & $\mathrm{XXX}$ & & & & XXX & \\
\hline L-9486 & & $\mathrm{XXX}$ & & & & $\mathrm{XXX}$ & & & \\
\hline N-9827 & & & & XXX & XXX & & & & \\
\hline G-7755 & & & & XXX & & $\mathrm{XXX}$ & & & \\
\hline
\end{tabular}

Tabela 2 - Avaliação do examinador 2 (ESSN).

\begin{tabular}{|c|c|c|c|c|c|c|c|c|c|}
\hline \multirow{2}{*}{ Paciente } & \multicolumn{4}{|c|}{ Pré-operatório } & \multicolumn{5}{|c|}{ Pós-operatório } \\
\hline & Leve & Moderado & Severo & $\begin{array}{l}\text { Muito } \\
\text { severo }\end{array}$ & Excelente & $\begin{array}{c}\text { Muito } \\
\text { bom }\end{array}$ & Bom & Satisfatório & Pobre \\
\hline P- 5171 & 1 & & & & & 3 & & & \\
\hline B -5532 & 2 & & & & & & & 7 & \\
\hline H-5694 & 6 & & & & & & 5,5 & & \\
\hline V-5822 & & & 7 & & & & 5.5 & & \\
\hline P-6110 & 1 & & & & & 3 & & & \\
\hline I-6538 & & & 10 & & & & & & \\
\hline G-6753 & & & 9 & & & & & 7.5 & \\
\hline M-7798 & & & 9 & & & & 5.0 & & \\
\hline P-8127 & 1 & & & & & 2 & & & \\
\hline J-8840 & & & 8 & & & & 5.5 & & \\
\hline Y-9107 & 3 & & & & & 3 & & & \\
\hline M-9281 & & & 7 & & & 2 & & & \\
\hline M-9415 & & & 9 & & & & & 8.0 & \\
\hline L-9486 & & 6 & & & & 3 & & & \\
\hline N-9827 & & & 9 & & & 2 & & & \\
\hline G-7755 & 11 & & & & & 3 & & & \\
\hline
\end{tabular}


Tabela 3 - Avaliação do examinador 3 (RSF).

\begin{tabular}{|c|c|c|c|c|c|c|c|c|c|}
\hline \multirow[b]{2}{*}{ Pacientes } & \multicolumn{4}{|c|}{ Pré-operatório } & \multicolumn{5}{|c|}{ Pós-operatório } \\
\hline & Leve & Moderado & Severo & $\begin{array}{l}\text { Muito } \\
\text { severo }\end{array}$ & Excelente & $\begin{array}{c}\text { Muito } \\
\text { bom }\end{array}$ & Bom & Satisfatório & Pobre \\
\hline P- 5171 & $\mathrm{XXX}$ & & & & & & $\mathrm{XXX}$ & & \\
\hline B -5532 & $\mathrm{XXX}$ & & & & & & & XXXX & \\
\hline H-5694 & XXX & & & & & & XXX & & \\
\hline V-5822 & & XXX & & & & & XXX & & \\
\hline B-5876 & $\mathrm{XXX}$ & & & & & & & & XXX \\
\hline P-6110 & XXX & & & & & $\mathrm{XXX}$ & & & \\
\hline I-6538 & & & $\mathrm{XXX}$ & & & & & & XXX \\
\hline G-6753 & & XXX & & & & & $\mathrm{XXX}$ & & \\
\hline M-7798 & & & $\mathrm{XXX}$ & & & $\mathrm{XXX}$ & & & \\
\hline P-8127 & & & & XXX & & & $\mathrm{XXX}$ & & \\
\hline W-8410 & XXX & & & & $\mathrm{XXX}$ & & & & \\
\hline $\mathrm{H}-8477$ & XXX & & & & $\mathrm{XXX}$ & & & & \\
\hline V-8586 & & & XXX & & & XXX & & & \\
\hline $\mathrm{J}-8840$ & & & & $\mathrm{XXX}$ & & & & & XXX \\
\hline Y-9107 & & & XXX & & & & & XXX & \\
\hline M-9281 & & XXX & & & & XXX & & & \\
\hline M-9415 & & & XXX & & & $\mathrm{XXX}$ & & & \\
\hline L-9486 & & & & $\mathrm{XXX}$ & & & & & $\mathrm{XXX}$ \\
\hline N-9827 & $\mathrm{XXX}$ & & & & & & & $\mathrm{XXX}$ & \\
\hline G-7755 & & & & XXX & & XXX & & & \\
\hline
\end{tabular}

Tabela 4 - Comparação dos resultados com tutor e sem tutor no pós-operatório (resumido).

\begin{tabular}{l|c|c|c|c|c|c}
\hline \multirow{2}{*}{ Pós } & & \multicolumn{2}{|c|}{ Com tutor } & \multicolumn{2}{c|}{ Sem tutor } & \multirow{2}{*}{ p-valor } \\
\cline { 3 - 6 } & & $\mathbf{N}$ & $\mathbf{\%}$ & $\mathbf{N}$ & $\mathbf{\%}$ & \\
\hline \multirow{3}{*}{ Examinador 1 } & Bom & 7 & $70 \%$ & 8 & $80 \%$ & \multirow{2}{*}{0,606} \\
\cline { 2 - 6 } & Ruim & 3 & $30 \%$ & 2 & $20 \%$ & \\
\hline \multirow{2}{*}{ Examinador 2 } & Bom & 7 & $70 \%$ & 6 & $60 \%$ & \multirow{2}{*}{0,639} \\
\cline { 2 - 6 } & Ruim & 3 & $30 \%$ & 4 & $40 \%$ & \\
\hline \multirow{2}{*}{ Examinador 3 } & Bom & 6 & $60 \%$ & 7 & $70 \%$ & \multirow{2}{*}{0,639} \\
\cline { 2 - 6 } & Ruim & 4 & $40 \%$ & 3 & $30 \%$ & \\
\hline \multirow{2}{*}{ Geral } & Bom & 20 & $66,7 \%$ & 21 & $70 \%$ & \multirow{2}{*}{0,781} \\
\cline { 2 - 6 } & Ruim & 10 & $33,3 \%$ & 9 & $30 \%$ & \\
\hline
\end{tabular}

previamente orientada e cuidadosa é extremamente simples. Nos casos em que fomos obrigados a reposicionar o modelador, atribuímos à falta de cuidados do responsável.

Enfim, mais estudos se fazem necessários, pois estamos tratando de uma patologia que se apresenta de múltiplas formas, atingindo a todas as raças e com alto poder de deformação.

\section{CONCLUSÃO}

No grupo de pacientes operados pela técnica de Göteborg, o uso do modelador nasal não apresentou resultados melhores estatisticamente significantes, quando comparado ao grupo de pacientes operados com a mesma técnica sem o uso do modelador nasal.

Não foi possível a avaliação funcional do nariz, o que associado ao pequeno número de pacientes avaliados torna 
necessária a realização de novos estudos, para melhor avaliação deste modelador.

\section{REFERÊNCIAS}

1. Buzzo CL. Tratamento cirúrgico da fissura labial pela técnica de Göteborg: seguimento de 7 anos. Rev Bras Cir Plást. 2010;25(2):251-9.

2. McCarthy GJ, Cutting CB, Hogan VM. Introduction to facial clefts. In: McCarthy JG, ed. Plastic surgery: cleft lip \& palate and craniofacial anomalies. Philadelphia: W.B. Saunders;1990. p.2437-50.

3. Figueiredo JCA, Freitas AG. Fissuras labiais. In: Mélega JM, ed. Cirurgia plástica: fundamentos e arte - cirurgia reparadora de cabeça e pescoço. Rio de Janeiro:Medsi;2002. p.89-109.

4. Millard Jr R. Unilateral cleft lip deformity. In: McCarthy JG, ed. Plastic surgery: cleft lip \& palate and craniofacial anomalies. Philadelphia: W.B. Saunders;1990. p.2627-51.
5. Anger J. Prof. Perseu Castro de Lemos e Prof. Victor Spina: a história da plástica em $\mathrm{Z}$ na queiloplastia para a correção das fissuras lábiopalatinas unilaterais. Rev Soc Bras Cir Plást. 2005;20(4):245-7.

6. Bonato Jr A. Fissura labiopalatal unilateral. In: Mélega JM, ed. Cirurgia plástica: fundamentos e arte - cirurgia reparadora de cabeça e pescoço. Rio de Janeiro:Medsi;2002. p.126-39.

7. McComb H. Primary correction of unilateral cleft lip nasal deformity: a 10-year review. Plast Reconstr Surg. 1985;75(6):791-9.

8. Nimi T, Natsume N, Tsukawaki T, Furukawa H, Masuda H, Kobayashi $\mathrm{M}$, et al. Treatment of the nostrils in patients with cleft lip by a nostril retainer. Br J Oral Maxillofac Surg. 2000;38(3):224-6.

9. Yuzuriha S, Matsuo K, Kondoh S. A newly designed nasal retainer to sustain the corrected shape of the nostril rim and anterior nasal recess for clef lip patients. Plast Reconstr Surg. 2001;108(2):452-5.

10. Anastassov Y, Chipkov C. Analysis of nasal and labial deformities in cleft lip, alveolus and palate patients by a new rating scale: preliminary report. J Craniomaxillofac Surg. 2003;31(5):299-303. 